\title{
Increased Performance in Elite Runners Following Individualized Timing of Sodium Bicarbonate Supplementation
}

\author{
Tue A.H. Lassen, Lars Lindstrøm, and Simon Lønbro \\ Aarhus University
}

\author{
Klavs Madsen \\ Norwegian School of Sport Sciences
}

\begin{abstract}
The present study investigated individualized sodium bicarbonate $\left(\mathrm{NaHCO}_{3}^{-}\right)$supplementation in elite orienteers and its effects on alkalosis and performance in a simulated sprint orienteering competition. Twenty-one Danish male and female elite orienteers (age $=25.2 \pm 3.6$ years, height $=176.4 \pm 10.9 \mathrm{~cm}$, body mass $=66.6 \pm 7.9 \mathrm{~kg}$ ) were tested twice in order to identify individual time to peak blood bicarbonate $\left(\mathrm{HCO}_{3}{ }^{-}\right.$peak) following supplementation of $0.3 \mathrm{~g} / \mathrm{kg}$ body mass $\mathrm{NaHCO}_{3}$ with and without warm-up. The athletes also performed two $3.5 \mathrm{~km}$ time-trial runs (TT-runs) following individualized timing of $\mathrm{NaHCO}_{3}$ supplementation (SBS) or placebo (PLA) on separate days in a randomized, double-blind, cross-over design. The occurrence of individual peak $\mathrm{HCO}_{3}{ }^{-}$and $\mathrm{pH}$ ranged from 60 to 180 min. Mean $\mathrm{HCO}_{3}{ }^{-}$and $\mathrm{pH}$ in SBS were significantly higher compared with PLA 10 min before and following the TT-run $(p<.01)$. SBS improved overall performance in the $3.5 \mathrm{~km}$ TT-run by 6 s compared with PLA $(775.5 \pm 16.2 \mathrm{~s}$ vs. $781.4 \pm 16.1 \mathrm{~s}$, respectively; $p<.05)$. SBS improved performance in the last half of the TT-run compared with PLA $(p<.01)$. In conclusion, supplementation with $\mathrm{NaHCO}_{3}$ followed by warm-up resulted in individualized alkalosis peaks ranging from 60 to $180 \mathrm{~min}$. Individualized timing of SBS in elite orienteers induced significant alkalosis before and after a $3.5 \mathrm{~km}$ TT and improved overall performance time by $6 \mathrm{~s}$, which occurred in the last half of the time trial. The present data show that the anaerobic buffer system is important for performance in these types of endurance events lasting $12-15$ min.
\end{abstract}

Keywords: acid-base balance, aerobic-anaerobic performance, ergogenic aids

The use of oxygen accounts for almost the entire turnover of ATP synthesis in long-term endurance exercise but the specific energetic metabolism during shorter endurance events (12-15 min) is less clear (Hargreaves \& Spriet, 2020). During this type of event, such as track-and-field running, sprint orienteering, and crosscountry skiing, the relative anaerobic versus aerobic contribution to total energy release will be small but important for performance since the ATP supply from nonmitochondrial sources may lead to increased hydrogen ion $\left(\mathrm{H}^{+}\right)$release and muscular acidosis.

In anaerobic exercise, the resynthesis of ATP for muscular work is initially covered by the PCr-ATP system, followed by glycolysis (Hargreaves \& Spriet, 2020). During anaerobic glycolysis, the cellular $\mathrm{H}^{+}$buffering capacity can be exceeded, resulting in a decrease in cellular $\mathrm{pH}$ associated with subsequent muscular fatigue (Fitts, 1994). This metabolic acidosis will have a negative impact on the release of calcium ions during muscle contraction, the activation of electrical signal receptors, the binding of calcium ions to troponin C, and metabolic enzyme activity (Fitts, 1994). However, it is unclear how much this system contributes during shorter (12-15 min) maximal aerobic and anaerobic exercise, and how significant it is for athlete performance.

During intense exercise, the accumulation of $\mathrm{H}^{+}$depends on various intra- and extracellular mechanisms buffering the $\mathrm{H}^{+}$ release. One important buffer system is the bicarbonate system and several studies show increased buffering capacity following anaerobic training (for a recent review, see Sahlin, 2014). Studies

Lassen, Lindstrøm, and Lønbro are with the Department of Public Health, Aarhus University, Aarhus, Midtjylland, Denmark. Madsen is with the Norwegian School of Sport Sciences, Oslo, Norway. Madsen (klavsm@nih.no) is corresponding author. also report that supplementation of bicarbonate $\left(\mathrm{HCO}_{3}^{-}\right)$and sodium ions $\left(\mathrm{Na}^{+}\right)$partly buffers the accelerated release of $\mathrm{H}^{+}$ associated with anaerobic activity, thereby lowering acidosis (Burke, 2013). Therefore, sodium bicarbonate supplementation (SBS) can be an indirect way to investigate the importance of the bicarbonate system for performance.

Several studies have investigated the acute effect of sodium bicarbonate $\left(\mathrm{NaHCO}_{3}\right)$ supplementation in various exercise activities, using subjects with different training status. There is a quite clear consensus that $\mathrm{SBS}$ will increase blood $\mathrm{HCO}_{3}{ }^{-}, \mathrm{pH}$, and lactate before and during several types of exercise (Heibel et al. 2018; Krustrup et al., 2015; Mueller et al., 2013). However, in regard to short-term endurance exercise, findings are conflicting, with the majority of studies showing no effect on performance (Hadzic et al., 2019).

Several facts could explain the divergent findings: (a) differences in the type of exercise and training status of the involved subjects; (b) the timing, amount, and type of supplement, which potentially can cause stomach problems and large individual variations in uptake and thus affect the outcome; and (c) the number of subjects, different performance tests, inconsistent use of preexercise, and even presupplementation warm-ups. These factors increase variation and probably reduce statistical power in studies where the possible effect on performance is quite small. Specifically, there is a large individual variability in time-to-peak $\mathrm{pH}$ after SBS, as shown by Sparks et al. (2017). Consequently, it is crucial to individualize the timing and to minimize the variability of the abovementioned factors when studying SBS and the influence on performance.

The type of exercise might be important when studying the effect of SBS. In sprint orienteering, athletes navigate an unmarked course using only a map and a compass while running over varied 
terrains for a period of around $15 \mathrm{~min}$. The sport is physically demanding with many changes of directions and accelerations. Although the running speed is varied, the heart rate is continuously close to maximum and studies show lactate concentrations of 8-12 mmol/L during competition (Hébert-Losier et al., 2015). Sprint orienteering is an event with very high aerobic demand and contribution of varied anaerobic energy production at different time periods. In that sense, sprint orienteering might be an interesting event when studying SBS and performance.

The primary aim of the present study was to investigate how individual timed SBS can influence performance in a field test designed to simulate sprint orienteering. The study was performed on elite athletes and designed as a randomized, double-blind, crossover design with sodium bicarbonate $\left(\mathrm{NaHCO}_{3}\right)$ or placebo (PLA) ingestion. We hypothesized that individualized SBS would improve performance and increase blood $\mathrm{HCO}_{3}{ }^{-}, \mathrm{pH}$, and lactate.

In order to investigate whether the bicarbonate system is important for performance in a 12-15 min intense endurance event, we conducted a study involving 21 elite athletes using individual timing of bicarbonate supplementation and a standard performance test with low variability. In addition, a specific $\mathrm{NaHCO}_{3}$ capsule product was given in order to avoid stomach problems, and we investigated whether warm-up would affect the bicarbonate timing. We also studied performance in a more applied but controlled setting, with a physical test almost identical to sprint orienteering.

\section{Methods}

\section{Participants}

Twenty-one elite orienteers (13 men, mean \pm SD; age $=26.4 \pm 3.3$ years, height $=181.8 \pm 9.6 \mathrm{~cm}$, and bodyweight $69.6 \pm 8.0 \mathrm{~kg}$; eight women, age $=23.0 \pm 3.5$ years, height $=167.6 \pm 6.3 \mathrm{~cm}$, and bodyweight $=61.7 \pm 4.9 \mathrm{~kg}$ ) volunteered to participate in the study. A total of 26 participants were recruited but five of them dropped out before or after the first test due to injuries. All participants met the following inclusion criteria: active athlete in the Danish National Centre for Elite Orienteering; aged between 18 and 40 years; nonsmoker; did not use any medication before or during participation (asthma medication allowed). The participants had between 10 and 25 years of experience in the sport of orienteering. They were recruited via the national team e-mail system with acceptance from the federation and national coaches. All participants were fully informed of any possible risks and discomforts associated with the experimental procedures before giving their written consent to participate. The study was performed in accordance with the Declaration of Helsinki and approved by the local
Scientific Ethics Committee of the Central Denmark Region (N-110-72-226-17).

\section{Experimental Protocol}

The pretest set-up consisted of a preliminary session including a familiarization with the time-trial run (TT-run). In addition, two tests to identify individual time to peak $\mathrm{HCO}_{3}{ }^{-}$blood bicarbonate $\left(\mathrm{HCO}_{3}{ }^{-}\right.$peak $)$with supplementation of $0.3 \mathrm{~g} / \mathrm{kg}$ body mass $\mathrm{NaHCO}_{3}$ were performed. One test was done with a warm-up, the other test without warm-up, and the two tests were separated by a minimum of $48 \mathrm{hr}$. A blood sample was obtained from a fingertip every 20 min until $\mathrm{HCO}_{3}^{-}$peak.

Following these tests, the athletes performed two $3.5 \mathrm{~km}$ TT-runs following individualized timing of SBS or PLA on two separate days in a randomized, double-blind, cross-over design (Figure 1). Blood samples were obtained from a fingertip before and after the TT-run.

The two TT-runs were separated by 3-7 days and were carried out under the same conditions and at the same time of day for each individual $( \pm 1 \mathrm{hr}$ ). For $48 \mathrm{hr}$ and $24 \mathrm{hr}$ before the first of the two TTruns, the participants were asked to report their training and food intake, respectively, and to replicate the training and diet before the subsequent TT-run. They were also instructed to avoid strenuous and prolonged exercise in the $48 \mathrm{hr}$ preceding the TT-run.

\section{Testing Procedures}

At the two pretests the participants ingested $0.3 \mathrm{~g} / \mathrm{kg}$ body mass $\mathrm{NaHCO}_{3}$. Blood levels of $\mathrm{HCO}_{3}{ }^{-}$and $\mathrm{pH}$ were measured every 20 min until $\mathrm{HCO}_{3}{ }^{-}$peak. In Pretest 1 , the participants were resting throughout the test, whereas in Pretest 2, the participants completed a low-intensity warm-up (60-70\% of $\left.\mathrm{HR}_{\max }\right) 40$ min before the point that corresponded to the time $\mathrm{HCO}_{3}{ }^{-}$peak occurred on Pretest 1 and continued until the peak level of $\mathrm{HCO}_{3}{ }^{-}$was registered.

Prior to each TT-run, the participants ingested either $0.3 \mathrm{~g} / \mathrm{kg}$ body mass $\mathrm{NaHCO}_{3}$ or $0.3 \mathrm{~g} / \mathrm{kg}$ body mass $\mathrm{CaCO}_{3}$. The supplementation was distributed using $575 \mathrm{mg}$ of content in enteric coated gelatin capsules to delay absorption of the supplementation to the intestine and thereby reduce gastrointestinal (GI) discomfort. The capsules were swallowed with water and in conjunction with eating a banana or an apple. The timing of the ingestion was determined individually for each participant based on the results of the pretest. The participants started their warm-up 40 min before the TT-run, following a standardized warm-up protocol.

The TT-runs were carried out as $3.5 \mathrm{~km}$ individual running tests involving 10 laps on a specially designed $350-\mathrm{m}$ route in an
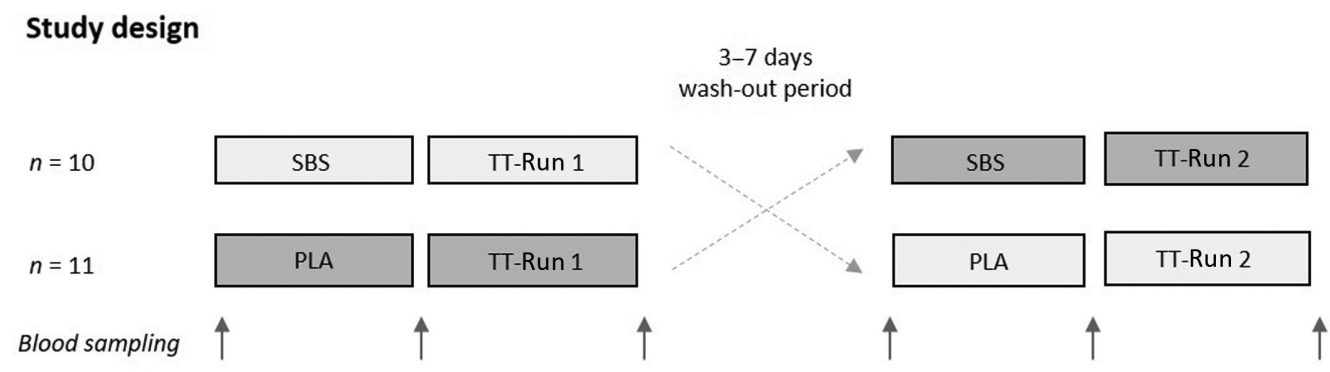

Figure 1 - Overview of the study design. The athletes performed two $3.5 \mathrm{~km}$ TT-runs following individualized timing of SBS or PLA on two separate days in a randomized, double-blind, cross-over design. TT-run = time-trial run; SBS = sodium bicarbonate supplementation; PLA = placebo. 


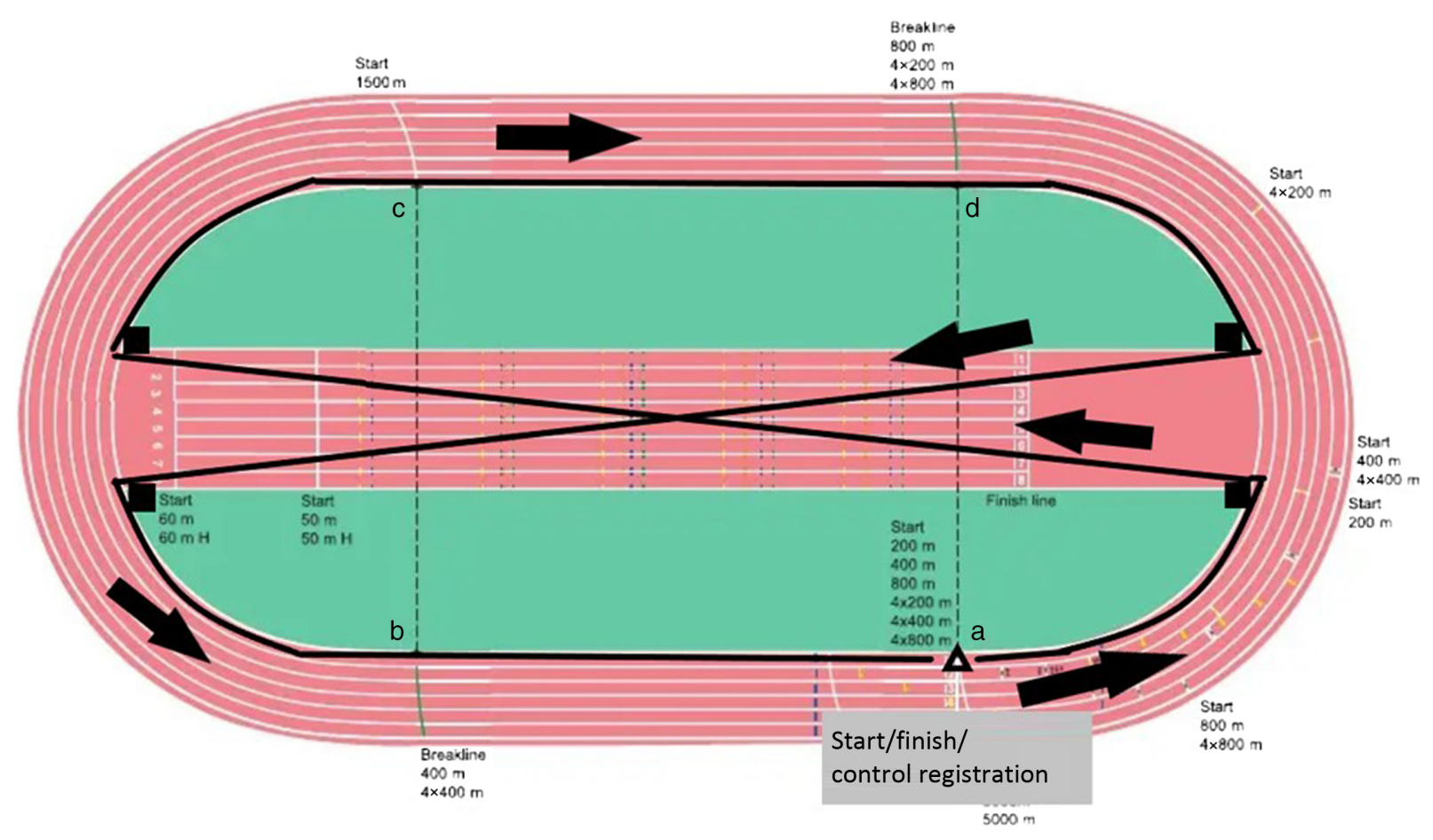

Figure 2 - Illustration of the randomized, controlled, cross-over study. All subjects performed two standardized 3.5 km TT-runs (TT-Run 1 and TT-Run 2) after individualized timing of either SBS or PLA. Pre and post SBS and placebo supplementation and immediately post TT-Runs 1 and 2, a fingertip blood sample was taken for lactate, $\mathrm{HCO}_{3}{ }^{-}$, and $\mathrm{pH}$ measurements. TT-runs = time-trial runs; $\mathrm{SBS}=$ sodium bicarbonate supplementation; PLA = placebo.

indoor running track area (see Figure 2). The route was designed to simulate the most common physical demands in a sprint-orienteering race. It consisted of four $90^{\circ}$ turns on each lap, two left turns and two right turns, and a control registration for every lap to simulate a complete stop and subsequent acceleration.

Ten minutes before, $1 \mathrm{~min}$ after, and $5 \mathrm{~min}$ after the TT-run, two blood samples were taken for lactate, $\mathrm{HCO}_{3}{ }^{-}$, and $\mathrm{pH}$ measurements. Before ingestion of $\mathrm{NaHCO}_{3}$ or PLA, 15 min before the TT-run and 2 min after the TT-run, the participants answered a questionnaire consisting of six questions on GI discomfort, where 0 was "no discomfort" and 20 was "worst imaginable discomfort." Fifteen minutes before and 2 min after the TT-run, the questionnaire also included one question to investigate whether the participants thought they had ingested $\mathrm{NaHCO}_{3}$ or PLA.

\section{Measurements}

All blood samples collected during the pretest and TT-run were taken from a fingertip into a capillary tube $(1 \mathrm{ml})$, and immediately analyzed for lactate (Biosen C-Line; EKF-diagnostic $\mathrm{GmbH}$, Magdeburg, Germany) or $\mathrm{HCO}_{3}{ }^{-}$and $\mathrm{pH}$ (ABL 800 Flex; Radiometer, Brønshøj, Denmark).

The timing of the TT-run with start, lap-splits, and finish was done with the most common timing system used in orienteering (SPORTident GmbH, Arnstadt, Germany).

\section{Statistics}

The study was planned to include approximately 20 participants in a randomized cross-over design. This number was based on an a priori sample size calculation with change in TT performance as the primary endpoint. The calculation was performed in STATA 16 (STATA Corp LP, TX) using an expected $1.7 \%$ increase $( \pm 1.7 \%$ $S D$ ) in performance following $\mathrm{NaHCO}_{3}$ ingestion (Carr et al., 2011), a $20 \%$ drop-out rate, $80 \%$ statistical power, and a $5 \%$ significance level.

Differences between SBS and PLA in TT-run performance, TT-run performance first half and second half, blood concentration of lactate, $\mathrm{HCO}_{3}{ }^{-}$and $\mathrm{pH}$, GI discomforts, and guesses regarding supplementation before and after the TT-run were analyzed using a paired-samples $t$ test. Differences between warm-up and no warm-up after SBS in $\mathrm{HCO}_{3}{ }^{-}$and $\mathrm{pH}$ were analyzed using a two-way analysis of variance with repeated measures (Group (With and Without Warm-up) $\times$ Time). Differences between SBS and PLA in TT-run lap-splits were analyzed using a two-way analysis of variance with repeated measures (Group $\times$ Time).

Statistical significance was accepted at $(p<.05)$. Data are presented as means \pm SEM unless stated otherwise.

\section{Results}

\section{Performance in the TT-Run}

There was a significant Group $\times$ Time interaction on performance in the TT-run $(p<.05$; Figure 3$)$. Overall performance improved by $\sim 6 \mathrm{~s}(775.5 \pm 16.2 \mathrm{~s}$ vs. $781.4 \pm 16.1 \mathrm{~s})$ following SBS compared with PLA (Table 1). In the first half of the TT-run, no significant difference between SBS and PLA was observed, while SBS improved performance in the last half of the TT-run compared with PLA $(p<.01 ;$ Table 1). 


\section{Blood and Plasma Metabolites in the TT-Run}

Blood lactate was $1.0 \pm 0.2$ and $1.0 \pm 0.2 \mathrm{mmol} / \mathrm{L} 10 \mathrm{~min}$ before the TT-run in SBS and PLA (Figure 4a). At 1 min and 5 min after the TT-run, blood lactate was $2.2 \mathrm{mmol} / \mathrm{L}$ and $2.0 \mathrm{mmol} / \mathrm{L}$ higher $(p<.01)$ in SBS $(11.0 \pm 3.0 .7 \mathrm{mmol} / \mathrm{L}$ and $9.2 \pm 0.5 \mathrm{mmol} / \mathrm{L})$ than in PLA $(8.8 \pm 0.5 \mathrm{mmol} / \mathrm{L}$ and $7.2 \pm 0.5 \mathrm{mmol} / \mathrm{L})$.

Mean $\mathrm{HCO}_{3}{ }^{-}$in SBS was higher $(p<.01)$ compared with PLA $10 \mathrm{~min}$ before the TT-run $(32.1 \pm 0.5 \mathrm{mmol} / \mathrm{L}$ vs. $27.0 \pm$ $0.3 \mathrm{mmol} / \mathrm{L}), 1 \mathrm{~min}$ after the TT-run $(19.4 \pm 0.7 \mathrm{mmol} / \mathrm{L}$ vs. $16.7 \pm$

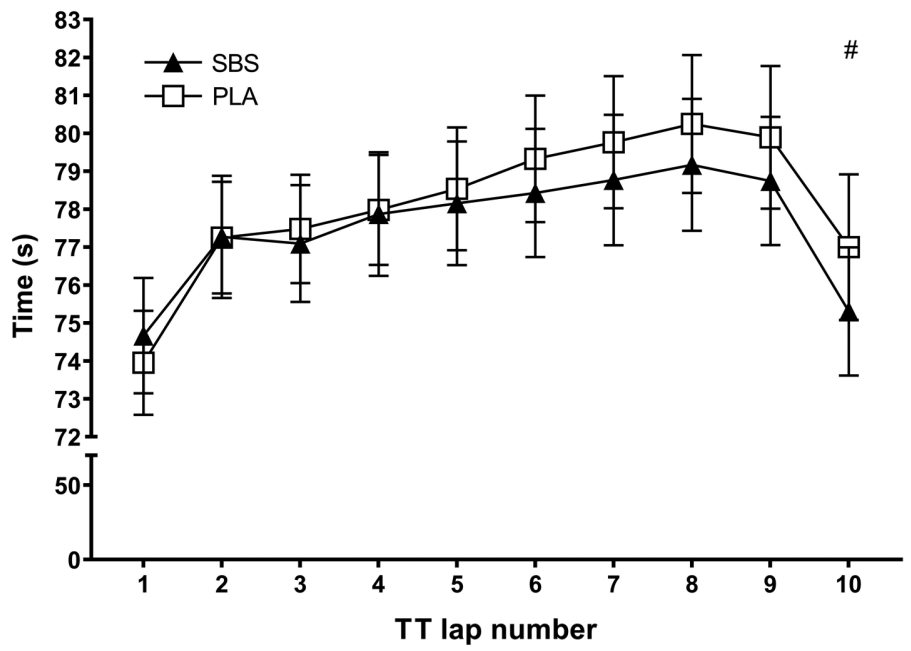

Figure 3 - Lap-splits during a $3.5 \mathrm{~km}$ TT-run following individualized timing of SBS or PLA. Values are presented as mean $\pm \mathrm{SEM} ; n=21$. TT-run $=$ time-trial run; $\mathrm{SBS}=$ sodium bicarbonate supplementation; PLA $=$ placebo. ${ }^{\#}$ Significant time and treatment effect $(p<.05)$.
$0.6 \mathrm{mmol} / \mathrm{L})$, and $5 \mathrm{~min}$ after the TT-run $(21.4 \pm 0.7 \mathrm{mmol} / \mathrm{L}$ vs $18.6 \pm 0.6 \mathrm{mmol} / \mathrm{L}$; Figure 4b).

Mean $\mathrm{pH}$ in SBS was higher $(p<.01)$ compared with PLA 10 min before the TT-run $(7.51 \pm 0.03$ vs. $7.45 \pm 0.03), 1$ min after the TT-run $(7.35 \pm 0.01$ vs. $7.30 \pm 0.01)$, and 5 min after the TT-run $(7.38 \pm 0.01$ vs. $7.32 \pm 0.01)$ (Figure $4 c$ ).

\section{The GI Discomfort and Guesses Regarding Supplementation in the TT-Run}

The means of the six questions regarding GI discomfort (Table 2) were not different between SBS and PLA before ingestion $(2.2 \pm 0.3$ vs. $2.2 \pm 0.3), 15 \mathrm{~min}$ before the TT-run $(4.1 \pm 0.4$ vs. $3.9 \pm 0.5)$, and 2 min after the TT-run $(3.5 \pm 0.6$ vs. $3.8 \pm 0.6)$.

The participants' guesses regarding supplementation (Figure 5) were not different in SBS and PLA $15 \mathrm{~min}$ before the TT-run $(7.6 \pm 1.1$ vs. $11.1 \pm 1.0)$ and $2 \mathrm{~min}$ after the TT-run $(8.9 \pm 1.3$ vs. $11.6 \pm 1.2)$.

\section{Blood Metabolites With and Without Warm-Up}

The analysis of variance revealed a significant increase over time in blood $\mathrm{pH}$ and $\mathrm{HCO}_{3}{ }^{-}$following both SBS and PLA with and without warm-up (Figure $6 ; p<.001$ ). There was no significant Group $\times$ Time interaction for $\mathrm{HCO}_{3}{ }^{-}$or $\mathrm{pH}$ concentration; however, a tendency toward a Group $\times$ Time interaction was indicated for $\mathrm{pH}(p=.09)$.

\section{Discussion}

To the best of our knowledge, this study is the first to investigate how individual timed SBS can affect performance in a $12-15 \mathrm{~min}$ intense endurance event. The most notable finding of this study is

Table 1 Performance Data for a 3.5 km TT-Run Following Individualized Timing of SBS or PLA

\begin{tabular}{lccc}
\hline Trials & Total running time & Time for the first half & Time for the second half \\
\hline SBS (s) & $775.5 \pm 16.2^{*}$ & $385.1 \pm 7.8$ & $390.4 \pm 7.3^{* *}$ \\
PLA (s) & $781.4 \pm 16.1$ & $385.2 \pm 8.5$ & $396.2 \pm 9.0$ \\
\hline
\end{tabular}

Note. Values are presented as mean $\pm \mathrm{SEM} ; n=21$. SBS $=$ sodium bicarbonate supplementation; PLA = placebo; TT-run = time-trial run.

Significant treatment effect: ${ }^{*} p<.05 ;{ }^{* *} p<.01$.
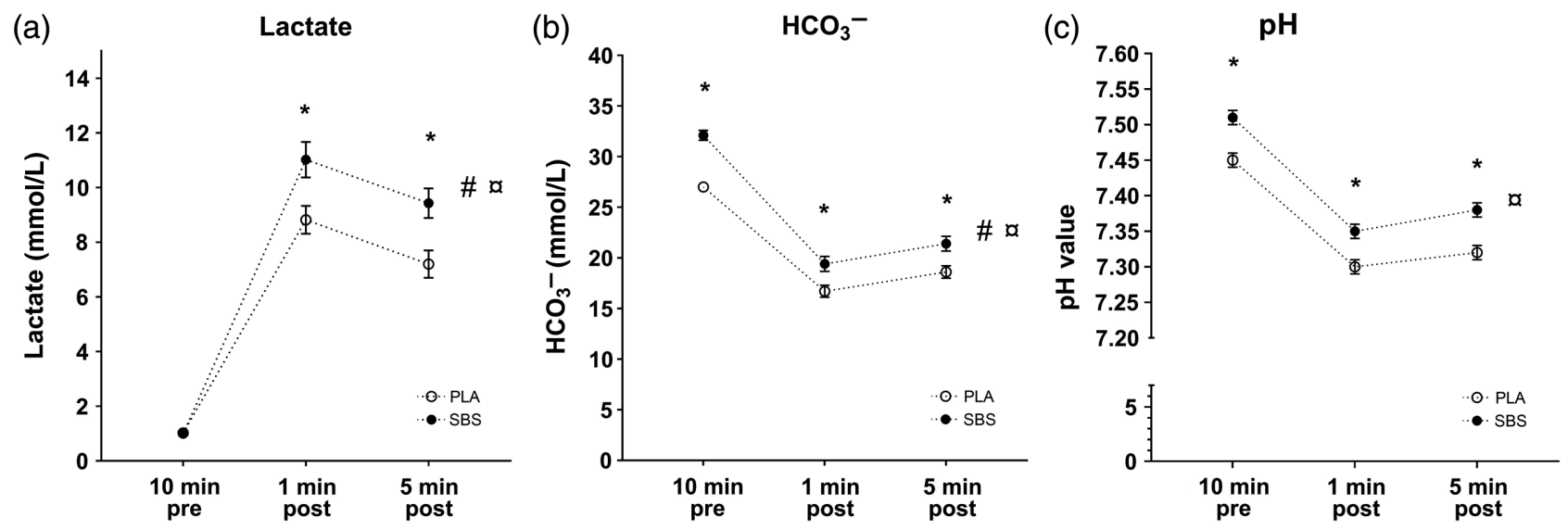

Figure 4 - (a) Blood lactate, (b) $\mathrm{HCO}_{3}{ }^{-}$, and (c) $\mathrm{pH}$ following individualized timing of SBS or PLA. Values are presented as mean \pm SEM; $n=21$. $\mathrm{SBS}=$ sodium bicarbonate supplementation; PLA = placebo. ${ }^{*}$ Significant treatment effect $(p<.01)$. 
Table 2 The GI Discomfort Response Before and After a $3.5 \mathrm{~km}$ TT-Run Following Individualized Timing of SBS or PLA

\begin{tabular}{lccc}
\hline Trials & Before intake & $\mathbf{1 5}$ min before start of the TT-run & $\mathbf{2}$ min after the TT-run \\
\hline SBS & $2.2 \pm 0.3$ & $4.1 \pm 0.4$ & $3.5 \pm 0.6$ \\
PLA & $2.2 \pm 0.3$ & $3.9 \pm 0.5$ & $3.8 \pm 0.6$ \\
\hline
\end{tabular}

Note. The participants answered six questions about GI discomforts on a scale of 0-20, ranging from 0 (no discomfort) to 20 (worst imaginable discomfort). Means of the six questions are presented. Values are presented means $\pm \mathrm{SEM} ; n=21$. No significant differences were observed. GI $=$ gastrointestinal; SBS $=$ sodium bicarbonate supplementation; PLA = placebo; TT-run = time-trial runs.

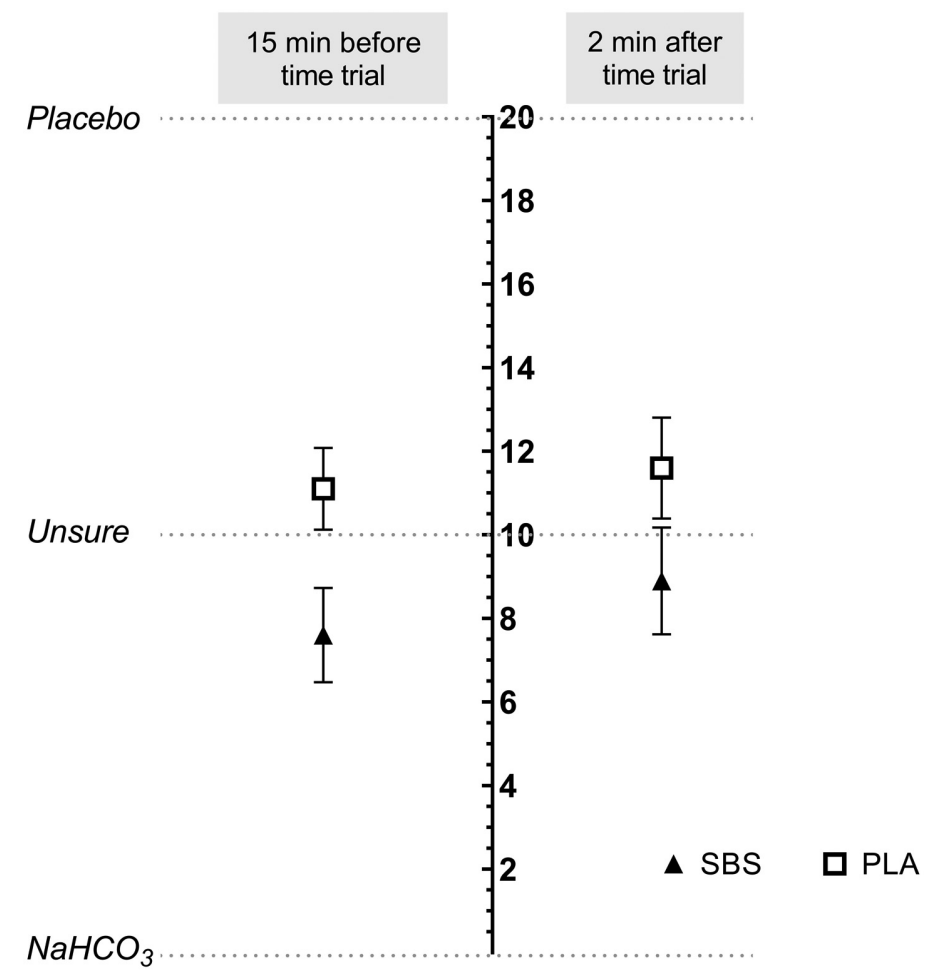

Figure 5 - The athletes' guesses regarding SBS or PLA, 15 min before and 2 min following the time-trial test. The question was "How sure are you if you have had $\mathrm{NaHCO}_{3}$ or placebo on a scale of $0-20$, where 0 was 'I'm sure I've got $\mathrm{NaHCO}_{3}$,' 10 was 'unsure' and 20 was 'I'm sure I've got placebo." Values are mean $\pm \mathrm{SEM} ; n=21$. SBS = sodium bicarbonate supplementation; PLA = placebo.

that SBS improved overall performance by $6 \mathrm{~s}$ in a $3.5 \mathrm{~km}$ TT-run compared with PLA. In addition, the associated changes in blood and plasma metabolite values indicate that exogenous $\mathrm{NaHCO}_{3}$ may reduce exercise-induced metabolic acidosis. As a consequence, the findings highlight that anaerobic metabolism and the bicarbonate buffering system are important for performance in these types of endurance events.

The current study shows high blood lactate values at exhaustion and a clear and significant effect of individualized SBS. There are no other available data from studies investigating the effect of SBS on running for 12-15 min. Comparing our results with the existing literature, our data are in line with a few other studies investigating the effect of SBS on physical activity lasting longer than 10 min (Egger et al., 2014; Marriott et al., 2015; Mueller et al., 2013), but in contrast with several other studies showing no effect (Afman et al., 2014; Freis et al., 2017; Tan et al., 2010). The study used a substantially greater number of participants than many previous studies (for a recent review, see Grgic et al., 2020) and

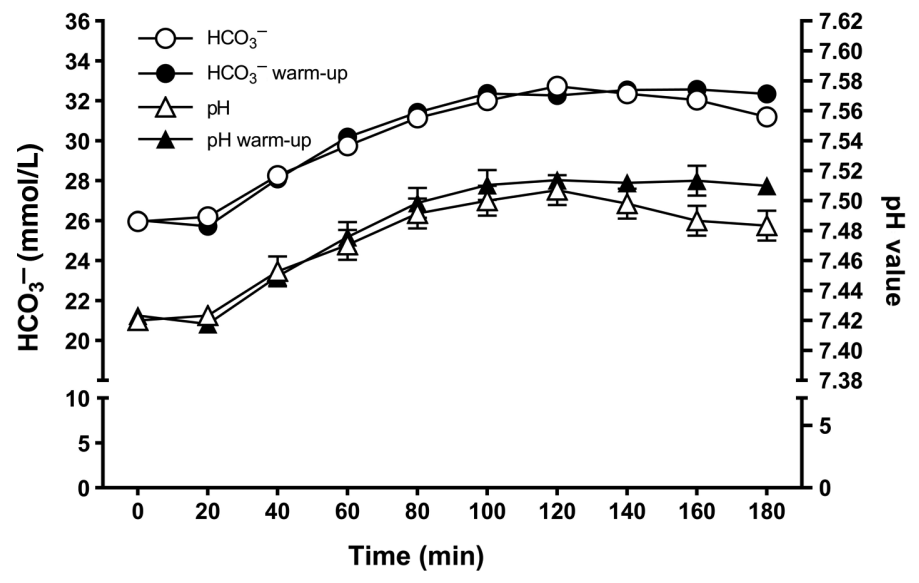

Figure $6-$ Blood $\mathrm{HCO}_{3}{ }^{-}$and blood $\mathrm{pH}$ following supplementation of $0.3 \mathrm{~g} / \mathrm{kg}$ body mass $\mathrm{NaHCO}_{3}$ with and without warm-up. Values are presented as mean \pm SEM; $n=21$. No significant time and treatment effects were observed.

a performance test that the participating subjects were very familiar with. In addition, the study used individualized timing of supplementation in a homogeneous group of highly trained athletes where the variations in performance tests and timing are usually small (Gough et al., 2017). With this setup, power calculations indicate that the present study would be able to detect significant changes in performance of around $0.5-0.7 \%$ or more.

The mean improvement of $5.9 \pm 10.7 \mathrm{~s}$ equates to a $0.8 \% \pm 1.4 \%$ increase in exercise performance. This is less than in several other studies showing improvements with SBS (Hadzic et al., 2019), and less than the predicted levels of improvement from the meta-analysis of Carr et al. (2011), which indicated a performance enhancement of $1.7 \%$. However, the present type of exercise has a larger contribution of aerobic energy consumption compared with several of these studies, and thereby is less dependent on anaerobic metabolism. Most importantly, the $\sim 6 \mathrm{~s}$ improvement is a significant and decisive improvement in elite sprint orienteering competitions.

The observed improvement in overall performance was generated in the second half of the TT-run, with the fifth to 10th lapsplits being significantly faster in the SBS TT-run compared with the PLA TT-run. This is in line with previous studies (Bishop \& Claudius, 2005; Hobson et al., 2014; Mero et al., 2004) and in line with the suggested effect for SBS in improving extracellular buffering and subsequently increasing intramuscular $\mathrm{pH}$ during the exercise period (Hargreaves \& Spriet, 2020).

In order to investigate the effect of SBS on performance, one needs to consider the large individual variation in uptake from the GI tract and how the blood, liver, and other organs treat the 
increased blood $\mathrm{HCO}_{3}{ }^{-}$. A previous study by Sparks et al. (2017), showed that mean time-to-peak $\mathrm{pH}$ was $64 \pm 19 \mathrm{~min}$ with a range of 10-85 min and a coefficient of variation of $29 \%$. In the present study, we determined individual time to peak blood $\mathrm{HCO}_{3}{ }^{-}$with a supplementation rate of $0.3 \mathrm{~g} / \mathrm{kg}$ body mass $\mathrm{NaHCO}_{3}$ and got similar results. These data indicate that one should determine individual time-to-peak $\mathrm{pH} / \mathrm{HCO}_{3}{ }^{-}$when investigating the effect of supplementation on performance in order to minimize the variation. Similarly, athletes using supplementation should be aware of their own time-to-peak in order to benefit most from the added buffering capacity.

In addition to individual timing, we also tested whether a moderate warm-up would influence blood $\mathrm{pH}$ and $\mathrm{HCO}_{3}{ }^{-}$. This is a typical competition situation and therefore an important aspect to take into consideration. The data for both blood $\mathrm{pH}$ and $\mathrm{HCO}_{3}{ }^{-}$ were very similar in the pretests with and without warm-up, indicating that moderate exercise for 40-100 $\mathrm{min}$ has no influence on the peak values for $\mathrm{pH}$ and $\mathrm{HCO}_{3}{ }^{-}$. Therefore, the SBS effect will not be reduced by a moderate warm-up, and a moderate warm-up will not affect the time-to-peak for blood $\mathrm{pH}$ and $\mathrm{HCO}_{3}{ }^{-}$.

Most studies show that SBS improves energy metabolism and ionic regulation (Egger et al., 2014; Heibel et al., 2018; Hilton et al., 2020; Stöggl et al., 2014). The significant elevation of blood $\mathrm{pH}$ and $\mathrm{HCO}^{-}$concentration following SBS in the pretest and TTrun were of similar magnitude compared with these studies. The exercise-induced declines in both values were quite similar in the two TT-runs but levels remained higher in the SBS TT-runs compared with the PLA TT-runs. It should be noted that blood $\mathrm{HCO}_{3}{ }^{-}$is only a part of the total buffer capacity in muscle and blood, but the data show a large effect of SBS on the blood pH system before and after intense exercise.

The present data on blood $\mathrm{pH}, \mathrm{HCO}_{3}{ }^{-}$, and lactate clearly indicate an improved buffering capacity in muscle and blood following SBS. Increased $\mathrm{H}^{+}$has been associated with fatigue at various steps within the excitation-contraction pathway, where the effect of acidosis will limit the ability to maintain submaximal output, although the exact mechanism is still unclear (Cheng et al., 2018). As seen from the lap split during the TT-run, the fatigue occurring in the last part of the TT-run could partly be due to intracellular acidosis and the reduced acidosis in the SBS TT-run may enable the maintenance of submaximal output for a longer period.

Traditional SBS has reported low to severe GI symptoms (Burke \& Pyne, 2007; Kahle et al., 2013). A relatively new product, enteric-coated SB, can attenuate GI symptoms following acute bicarbonate loading (Hilton et al., 2019). Delayed release of SB has been shown to reduce the incidence and severity of GI symptoms compared with an aqueous solution, without affecting the time-topeak blood $\left[\mathrm{HCO}^{-}\right]$and $\mathrm{pH}$. On the other hand, changes in blood $\left[\mathrm{HCO}^{-}\right]$and $\mathrm{pH}$ were lower with enteric-coated SB, potentially given less effect from the supplementation, but a recent study using enteric-coated SB showed an improvement in high-intensity cycling performance in trained cyclists compared with PLA (Hilton et al., 2020). In the present study, we used enteric-coated SB and the subjective reports from the subjects showed no differences in GI discomfort before and following the TT-run (Table 2). In line with this, it was difficult for the subjects to guess whether they had PLA or SBS (Figure 5).

The strengths of the present study are the use of a quite large homogenous group of experienced runners that are familiar with the used performance test. In addition, the individual timing of the supplement limits the variation and the $\mathrm{NaHCO}_{3}$ capsule product minimized stomach problems. The main limitation is the narrow perspective with this type of event and duration of the competition. The practical implication of these findings is that the anaerobic buffer system is crucial for performance in this type of events. Coaches and athletes should take this into consideration in their training planning and use specific training models which aim to improve the muscle and blood buffer system.

In conclusion, this study is the first to demonstrate that individual timing of $0.3 \mathrm{~g} / \mathrm{kg}$ body mass of enteric-coated $\mathrm{NaHCO}_{3}$ improves high-intensity endurance running in a $3.5 \mathrm{~km}$ time trial. The induced alkalosis in the TT-run improved overall performance by $6 \mathrm{~s}$, which occurred in the last half of the TT-run. Supplementation with $\mathrm{NaHCO}_{3}$ resulted in individualized alkalosis peaks ranging between 60 and $180 \mathrm{~min}$ after administration, independent of a warm-up period. The present data show that the anaerobic buffer system is a minor but very important aspect of performance in sprint orienteering competitions and similar types of endurance events lasting 12-15 min.

\section{Acknowledgments}

The authors acknowledge the participation and dedication of the athletes on the Danish national orienteering team, which made it possible to conduct this study. In addition, they acknowledge the laboratory technicians (Gitte Kaiser Hartvigsen and Janni Mosgaard Jensen) for helping with the blood sampling and analyses. This work was financially supported by Team Denmark, which is an organization funded by the Danish government with the purpose of promoting elite sports in Denmark. All authors designed the study. Data were collected and analyzed by T.A.H. Lassen, L. Lindstrøm, and S. Lønbro. All authors interpreted the data and prepared the manuscript. All authors approved the final version of the paper. The authors declare no conflicts of interest.

\section{References}

Afman, G., Garside, R.M., Dinan, N., Gant, N., Betts, J.A., \& Williams, C. (2014). Effect of carbohydrate or sodium bicarbonate ingestion on performance during a validated basketball simulation test. International Journal of Sport Nutrition and Exercise Metabolism, 24(6), 632-644. PubMed ID: 24901305 doi:10.1123/ijsnem.2013-0168

Bishop, D., \& Claudius, B. (2005). Effects of induced metabolic alkalosis on prolonged intermittent-sprint performance. Medicine and Science in Sports and Exercise, 37(5), 759-767. PubMed ID: 15870629 doi:10.1249/01.MSS.0000161803.44656.3C

Burke, L.M. (2013). Practical considerations for bicarbonate loading and sports performance. Nestle Nutrition Institute Workshop Series, 75, 15-26. doi:10.1159/000345814

Burke, L.M., \& Pyne, D.B. (2007). Bicarbonate loading to enhance training and competitive performance. International Journal of Sports Physiology and Performance, 2(1), 93-97. PubMed ID: 19255457 doi:10.1123/ijspp.2.1.93

Carr, A.J., Hopkins, W.G., \& Gore, C.J. (2011). Effects of acute alkalosis and acidosis on performance: A meta-analysis. Sports Medicine, 41(10), 801-814. PubMed ID: 21923200 doi:10.2165/11591440000000000-00000

Cheng, A.J., Place, N., \& Westerblad, H. (2018). Molecular basis for exercise-induced fatigue: The importance of strictly controlled cellular Ca 2+ handling. Cold Spring Harbor Perspectives in Medicine, 8(2). doi:10.1101/cshperspect.a029710 
Egger, F., Meyer, T., Such, U., \& Hecksteden, A. (2014). Effects of sodium bicarbonate on high-intensity endurance performance in cyclists: A double-blind, randomized cross-over trial. PLoS One, 9(12), e114729. PubMed ID: 25494054 doi:10.1371/journal.pone. 0114729

Fitts, R.H. (1994). Cellular mechanisms of muscle fatigue. Physiological Reviews, 74(1), 49-94. PubMed ID: 8295935 doi:10.1152/physrev. 1994.74.1.49

Freis, T., Hecksteden, A., Such, U., \& Meyer, T. (2017). Effect of sodium bicarbonate on prolonged running performance: A randomized, double-blind, cross-over study. PLoS One, 12(8), e0182158. PubMed ID: 28797049 doi:10.1371/journal.pone.0182158

Gough, L.A., Deb, S.K., Sparks, A., \& McNaughton, L.R. (2017). The reproducibility of 4-km Time Trial (TT) performance following individualised sodium bicarbonate supplementation: A randomised controlled trial in trained cyclists. Sports Medicine - Open, 3(1), 34. PubMed ID: 28936625 doi:10.1186/s40798-017-0101-4

Grgic, J., Rodriguez, R.F., Garofolini, A., Saunders, B., Bishop, D.J., Schoenfeld, B.J., \& Pedisic, Z. (2020). Effects of sodium bicarbonate supplementation on muscular strength and endurance: A systematic review and meta-analysis. Sports Medicine, 50(7), 1361-1375. doi:10.1007/s40279-020-01275-y

Hadzic, M., Eckstein, M.L., \& Schugardt, M. (2019). The impact of sodium bicarbonate on performance in response to exercise duration in athletes: A systematic review. Journal of Sports Science and Medicine, 18(2), 271-281. PubMed ID: 31191097 doi:10.25932/ publishup-42807

Hargreaves, M., \& Spriet, L.L. (2020). Skeletal muscle energy metabolism during exercise. Nature Metabolism, 2(9), 817-828. PubMed ID: 32747792 doi:10.1038/s42255-020-0251-4

Hébert-Losier, K., Platt, S., \& Hopkins, W.G. (2015). Sources of variability in performance times at the world orienteering championships. Medicine \& Science in Sports \& Exercise, 47(7), 1523-1530. doi:10. 1249/mss.0000000000000558

Heibel, A.B., Perim, P.H.L., Oliveira, L.F., McNaughton, L.R., \& Saunders, B. (2018). Time to optimize supplementation: Modifying factors influencing the individual responses to extracellular buffering agents. Frontiers in Nutrition, 5, 35. doi:10.3389/fnut.2018. 00035

Hilton, N.P., Leach, N.K., Hilton, M.M., Sparks, S.A., \& McNaughton, L.R. (2020). Enteric-coated sodium bicarbonate supplementation improves high-intensity cycling performance in trained cyclists. European Journal of Applied Physiology, 120(7), 1563-1573. doi:10.1007/s00421-020-04387-5

Hilton, N.P., Leach, N.K., Sparks, S.A., Gough, L.A., Craig, M.M., Deb, S.K., \& McNaughton, L.R. (2019). A novel ingestion strategy for sodium bicarbonate supplementation in a delayed-release form: A randomised crossover study in trained males. Sports Medicine -
Open, 5(1), 4. PubMed ID: 30680463 doi:10.1186/s40798-0190177-0

Hobson, R.M., Harris, R.C., Martin, D., Smith, P., Macklin, B., ElliottSale, K.J., \& Sale, C. (2014). Effect of sodium bicarbonate supplementation on 2000-m rowing performance. International Journal of Sports Physiology and Performance, 9(1), 139-144. PubMed ID: 23579002 doi:10.1123/ijspp.2013-0086

Kahle, L.E., Kelly, P.V., Eliot, K.A., \& Weiss, E.P. (2013). Acute sodium bicarbonate loading has negligible effects on resting and exercise blood pressure but causes gastrointestinal distress. Nutrition Research, 33(6), 479-486. PubMed ID: 23746564 doi:10.1016/j. nutres.2013.04.009

Krustrup, P., Ermidis, G., \& Mohr, M. (2015). Sodium bicarbonate intake improves high-intensity intermittent exercise performance in trained young men. Journal of the International Society of Sports Nutrition, 12(1), 25. doi:10.1186/s12970-015-0087-6

Marriott, M., Krustrup, P., \& Mohr, M. (2015). Ergogenic effects of caffeine and sodium bicarbonate supplementation on intermittent exercise performance preceded by intense arm cranking exercise. Journal of the International Society of Sports Nutrition, 12(1), 13. doi:10.1186/s12970-015-0075-x

Mero, A.A., Keskinen, K.L., Malvela, M.T., \& Sallinen, J.M. (2004). Combined creatine and sodium bicarbonate supplementation enhances interval swimming. Journal of Strength and Conditioning Research, 18(2), 306-310. PubMed ID: 15142001 doi:10.1519/r12912.1

Mueller, S.M., Gehrig, S.M., Frese, S., Wagner, C.A., Boutellier, U., \& Toigo, M. (2013). Multiday acute sodium bicarbonate intake improves endurance capacity and reduces acidosis in men. Journal of the International Society of Sports Nutrition, 10(1), 16. PubMed ID: 23531361 doi:10.1186/1550-2783-10-16

Sahlin, K. (2014). Muscle energetics during explosive activities and potential effects of nutrition and training. Sports Medicine, 44, 167-173). doi:10.1007/s40279-014-0256-9

Sparks, A., Williams, E., Robinson, A., Miller, P., Bentley, D.J., Bridge, C., \& Mc Naughton, L.R. (2017). Sodium bicarbonate ingestion and individual variability in time-to-peak $\mathrm{pH}$. Research in Sports Medicine, 25(1), 58-66. PubMed ID: 27934546 doi:10.1080/15438627. 2016.1258645

Stöggl, T., Torres-Peralta, R., Cetin, E., \& Nagasaki, M. (2014). Repeated high intensity bouts with long recovery: Are bicarbonate or carbohydrate supplements an option? Scientific World Journal, 2014, 145747. PubMed ID: 25431775 doi:10.1155/2014/145747

Tan, F., Polglaze, T., Cox, G., Dawson, B., Mujika, I., \& Clark, S. (2010). Effects of induced alkalosis on simulated match performance in elite female water polo players. International Journal of Sport Nutrition and Exercise Metabolism, 20(3), 198-205. PubMed ID: 20601737 doi:10.1123/ijsnem.20.3.198 